\title{
Searching for Dark Matter in Distorted Starlight
}

\author{
Statistical studies of the motions of millions of stars may reveal the subtle \\ imprint of dark matter.
}

By Sophia Chen

$\mathrm{R}$ esearchers hypothesize that as dark matter passes in front of a bright object, it should distort that object's

brightness, such that the object appears to move. However, because the amounts of dark matter are small on cosmological scales, the apparent motions are subtle, making it difficult to resolve this effect, known as weak astrometric gravitational lensing. Now, Siddharth Mishra-Sharma of New York University and colleagues detail a statistical framework that aggregates these subtle apparent changes in object motion. Their framework should be sensitive to the signatures predicted for several different dark matter theories.

The framework employs data gathered in astrometry measurements, which track the positions and motions of stars and galaxies. The researchers map the velocity and acceleration distributions of these objects as frequency spectra and find that their distributions vary depending on the assumed type of dark matter. The technique also allows them to use asymmetries in the distributions-introduced by the Sun's motion through the

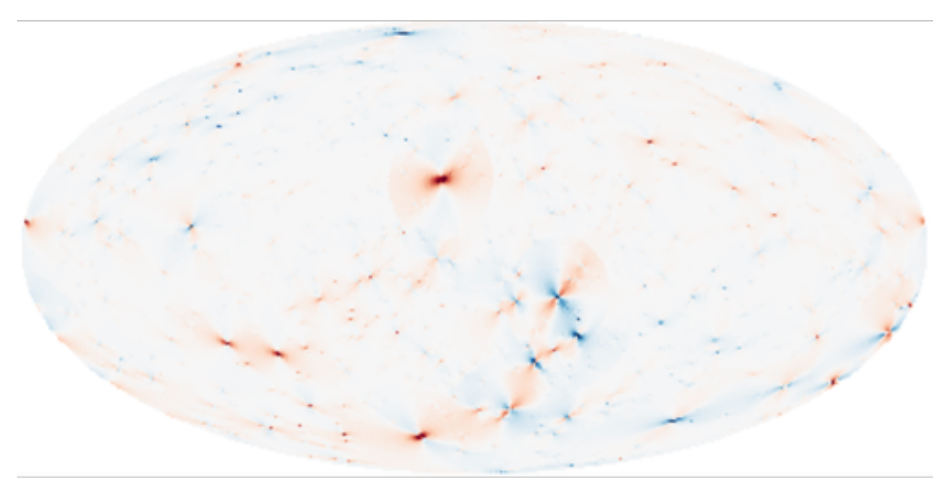

Credit: S. Mishra-Sharma et al., Phys. Rev. D (2020) 